\title{
Attenuating pandemic supply shocks with 3D printed emergency use equipment
}

\section{Karim I. Budhwani ( $\nabla$ IronMan@CerFlux.com )}

CerFlux, Inc. https://orcid.org/0000-0001-8823-1695

\author{
Albert T. Pierce \\ University of Alabama at Birmingham \\ Nitin Arora \\ University of Alabama at Birmingham
}

\section{Short Report}

Keywords: Additive manufacturing, Medical devices, Personal protective equipment, COVID-19, Biomedical technology, 3D printing, Policy

Posted Date: January 5th, 2022

DOI: https://doi.org/10.21203/rs.3.rs-1229836/v1

License: @ (i) This work is licensed under a Creative Commons Attribution 4.0 International License. Read Full License 


\section{Attenuating pandemic supply shocks with 3D printed emergency use equipment}

Karim I. Budhwani ${ }^{1,2 *}$, Albert T. Pierce ${ }^{2}$, Nitin Arora ${ }^{2}$

${ }^{1}$ CerFlux, Inc., Birmingham, AL 35205, USA.

2University of Alabama at Birmingham (UAB), Birmingham, AL 35294, USA.

KEYWORDS:

Additive manufacturing; Medical devices; Personal protective equipment; COVID-19; Biomedical technology; 3D printing; Policy.

WORD COUNT: 800 


\section{Abstract}

The fast-moving COVID-19 pandemic stressed supply chains even for some of the best prepared medical facilities. As a result, producing on-demand personal protective equipment (PPE) using additive manufacturing (AM) capabilities (3D-printing) emerged as a common strategy. While layer-by-layer processing confers several advantages to AM, it also imposes prohibitive print times, particularly for large volume parts. This leads to potential for using AM to rapidly produce smaller adaptors that modify available components for addressing critical shortages in emergencies. We applied this principle in developing a quick, simple, and low-cost adaptation of elastomeric half-mask respirators for emergency use in high-risk clinical settings. As we move toward reopening society, we must proactively build stronger bridges between engineering and medicine to respond to critical shortages in PPE ensuing from a potential second wave.

\section{Introduction}

Reconfiguring industrial masks for clinical settings "evoke Darth Vader, but these masks may save your doctor's life" observed the New York Times. ${ }^{1}$ In the absence of a coordinated national response, local institutions ${ }^{2}$ were left with no choice but to step up and address critical shortages in personal protective equipment (PPE) due to the abrupt distress in supply chains ${ }^{3,4}$ from the fast-moving COVID-19 pandemic. Much of this centered around producing on-demand PPE using additive manufacturing (AM) capabilities (3D-printing).

The choice of AM for such emergency response was not accidental. AM inherently supports fabricating a wide range of complex geometries ${ }^{5}$ using relatively low-cost, universally available 3D printers with minimal material waste. These are further enhanced, by crowd sourced designs that are easy to customize for local contexts and share around the world, including resource poor settings, via platforms like the NIH 3D Print Exchange. ${ }^{6}$ It is evident that were it not for AM, critical shortages in PPE would have resulted in further devastating consequences of the pandemic.

However, the layer-by-layer processing which confers several advantages to AM, also induces some weaknesses including processing times ${ }^{7}$ particularly for large volume parts. This leads to potential for using AM to rapidly produce smaller adaptors that modify available components for addressing critical shortages in emergencies. We applied this principle in developing a quick, simple, and low-cost adaptation of elastomeric half-mask respirators for emergency use in high-risk clinical settings.

\section{Methods}

In responding to COVID-19 supply shocks, we designed the Pierce-Arora-Budhwani Respirator (PABR), a simple mechanism to leverage existing 3D printing capacity to modify EHMRs into reusable N100 respirators. The 3D printed adaptors are easy to make and use, and consume less than $\$ 2$ in materials, making them accessible in resource poor settings. A detailed step-by-step guide for producing PABRs and related creative commons-noncommercial editable design files are available at the NIH 3D Print Exchange. ${ }^{6}$

Sealing properties were tested with a Powerbuilt vacuum pump (model 648744) by applying supramaximal vacuum (0.52 bar vacuum) from inside the PABR as well as outside. Qualitative fit test was performed in accordance with OSHA standards for hospital employees using the bitter test agent. Quantitative fit tests were performed using standards for half mask respirators. OSHA has determined that the controlled negative pressure (CNP) fit factor must be a minimum of 100 which approximately correspond to 1,000 for Ambient Aerosol (AA) or Nuclei Counting (NC). 


\section{Results}

PABRs could withstand more than five times maximal inspiratory pressure. Applied vacuum readily held for more than 2 minutes on each side. Qualitative bitter-agent tests use the person's sense of taste or smell, or reaction to an irritant. This is a pass/fail method that relies on whether or not a leak of the test substance is detected while wearing the face piece. On the other hand, CNP fit tests, based on generating and maintaining a constant negative pressure in the respirator, provide a quantitative measure of the facepiece fit for negative pressure respirators. PABRs passed qualitative bitter-agent tests and outperformed full-face counterparts in CNP quantitative test (Figure 1) performed in accordance with OSHA standards for hospital employees.

(A)

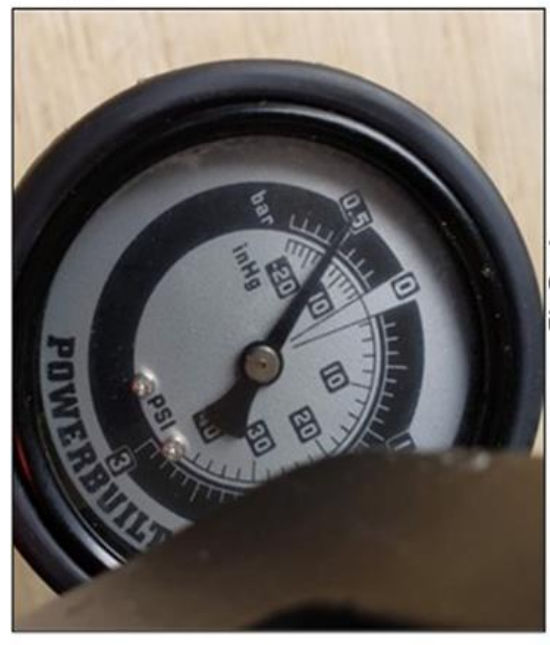

(B)

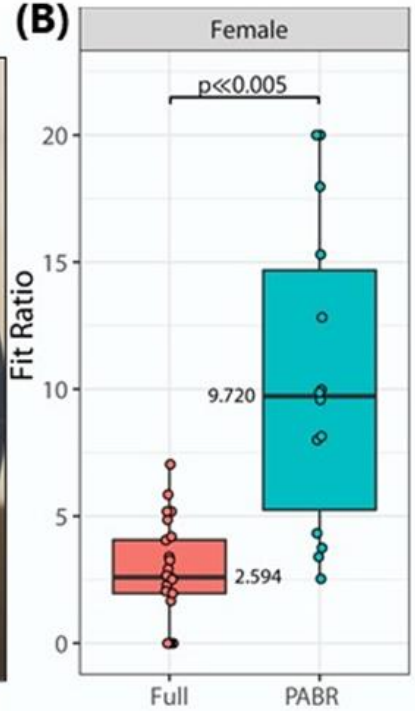

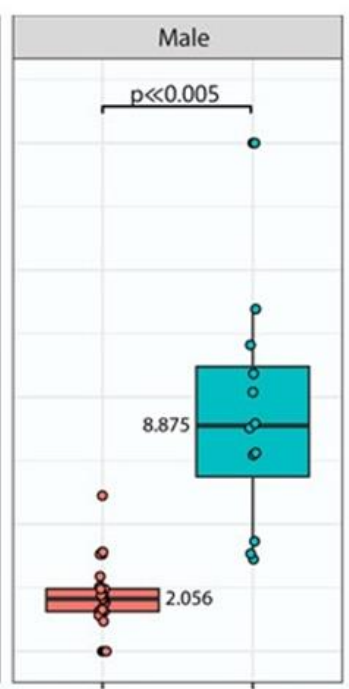

Füll

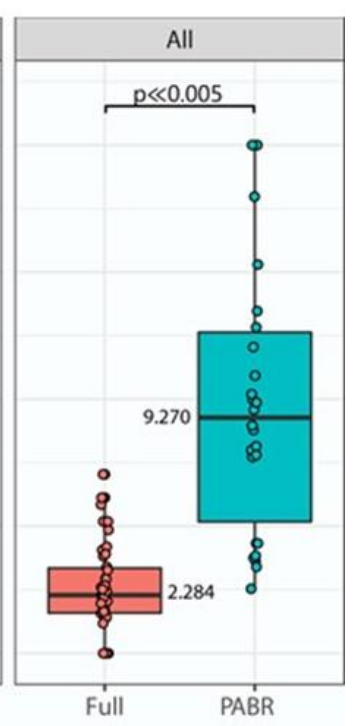

Figure 1. Testing. (A) PABR could withstand 0.52 bar vacuum which is more than five times maximal inspiratory pressure. (B) PABRs outperformed full face counterparts in terms of fit factors relative to OSHA determined minimum fit factors for that class (Welch's t-test $t(27.74)=6.8239, p=1.078 \mathrm{e}-07$ ).

\section{Discussion}

Elastomeric respirators are cost-effective, reusable PPE that are designed for appropriate facial seal, filtration, prolonged wear, and decreased resistance to breathing. These are available in full-face and half-mask configurations; each has its own benefits and liabilities. Elastomeric fullface masks provide eye protection; however, they also cause distortion and significant limitation of view, may suffer from view fogging, cannot be appropriately sealed to the face while wearing glasses, and are more expensive. Although elastomeric half-mask respirators (EHMR) do not provide eye protection, they are conducive for integration with covering specifically designed for eyes. Moreover, EHMRs do not cause view distortion, create only slight view obstruction with downward gaze, prevent view fogging of protective eyewear, can be sealed to the face while wearing prescription glasses, and are typically less expensive.

While there are numerous certified EHMRs on the market, there is significant concern that respirator cartridges for these cannot be adequately decontaminated after use in healthcare settings. Since EHMR decontamination is non-negotiable when used as COVID-19 PPE, disinfection of every component of EHMRs is critical, requiring cartridges to be discarded frequently. These are expensive and susceptible to supply shocks.

The PABR is one example of producing emergency use PPE by modifying available components using AM with locally sourced materials. As we move toward reopening society, 
infection risk will increase for healthcare professionals on the frontlines which in turn will increase PPE demand ${ }^{8,9}$ and possibly lead to critical shortages of the same. We must proactively build stronger bridges between engineering and medicine to respond to critical shortages in PPE ensuing from a potential second wave. Such bridges may indeed evoke Darth Vader, but a kinder and gentler Vader, focused on saving the lives of everyone around them.

Be safe and may the force be with you.

\section{References}

1. Hamby C. They evoke Darth Vader, but these masks may save your doctor's life [Internet]. New York Times. 2020;Available from: https://www.nytimes.com/2020/05/27/us/coronavirus-masks-elastomeric-respirators.html

2. Robinson F. Self-protection: how NHS doctors are sourcing their own PPE. BMJ [Internet] 2020;369(May):m1834. Available from: http://dx.doi.org/doi:10.1136/bmj.m1834

3. Ranney ML, Griffeth V, Jha AK. Critical Supply Shortages - The Need for Ventilators and Personal Protective Equipment during the Covid-19 Pandemic. N Engl J Med [Internet] 2020;NEJMp2006141. Available from: http://www.nejm.org/doi/10.1056/NEJMp2006141

4. Kamerow D. Covid-19: The crisis of personal protective equipment in the US. BMJ [Internet] 2020;369(April):2930. Available from: http://dx.doi.org/doi:10.1136/bmj.m1367

5. Ngo TD, Kashani A, Imbalzano G, Nguyen KTQ, Hui D. Additive manufacturing (3D printing): A review of materials, methods, applications and challenges. Compos Part B Eng [Internet] 2018;143(December 2017):17296. Available from: https://doi.org/10.1016/j.compositesb.2018.02.012

6. Pierce AT, Arora N, Budhwani KI. Pierce-Arora-Budhwani Respirator (PABR) [Internet]. NIH 3D Print Exch. 2020 [cited 2020 Jan 5];Available from: https://3dprint.nih.gov/discover/3dpx-014221

7. Wang X, Jiang M, Zhou Z, Gou J, Hui D. 3D printing of polymer matrix composites: A review and prospective. Compos Part B Eng [Internet] 2017;110:442-58. Available from: http://dx.doi.org/10.1016/j.compositesb.2016.11.034

8. lacobucci G. Covid-19: Doctors still at "considerable risk" from lack of PPE, BMA warns. BMJ [Internet] 2020;368(March):m1316. Available from: http://dx.doi.org/doi:10.1136/bmj.m1316

9. Shepherd A. Covid-19: Frontline doctors continue PPE fight. BMJ [Internet] 2020;369(June):m2188. Available from: http://www.ncbi.nlm.nih.gov/pubmed/32487701

\section{Author contributions}

The manuscript was written through contributions of all authors. All authors have given approval to the final version of the manuscript. Authors declare no competing interests.

\section{Additional information}

1. Step-by-step PABR fabrication guide

2. Creative commons-noncommercial editable design files for PABR components available at the NIH 3D Print Exchange in Autodesk® Fusion 360, Step, and STL format:

a. Clamp: https://3dprint.nih.gov/discover/3DPX-014221

b. Bayonet: https://3dprint.nih.gov/discover/3DPX-014434

3. Ultimaker $3 \mathrm{mf}$ project files with $3 \mathrm{D}$ complete print settings also included. 